\title{
Controlling the state of quantum spins with electric currents
}

\author{
Sebastian Loth ${ }^{1}$, Kirsten von Bergmann ${ }^{1,2}$, Markus Ternes ${ }^{1,3}$, Alexander F. Otte ${ }^{1,4}$, \\ Christopher P. Lutz ${ }^{1}$ and Andreas J. Heinrich ${ }^{1 \star}$
}

\begin{abstract}
A current of spin-polarized electrons senses and controls the magnetic state of nanostructured materials ${ }^{1}$. Obtaining similar electrical access to quantum spin systems, such as singlemolecule magnets, is still in its infancy ${ }^{2}$. Recent progress has been achieved by probing the spin system near thermal equilibrium $^{3-9}$. However, it is the elusive non-equilibrium properties of the excited states that govern the time evolution of such structures and will ultimately establish the feasibility of applications in data storage $\mathrm{e}^{2,10}$ and quantum information processing ${ }^{11,12}$. Here we use spin-polarized scanning tunnelling microscopy ${ }^{13}$ to pump electron spins of atoms on surfaces into highly excited states and sense the resulting spatial orientation of the spin. This electrical control culminates in complete inversion of the spin-state population and gives experimental access to the spin relaxation times of each excited state. The direction of current flow determines the orientation of the atom's spin, indicating that electrical switching and sensing of future magnetic bits is feasible in the quantum regime.
\end{abstract}

Atomic-scale magnetic structures consist of only a few strongly coupled electron spins. This results in well-separated quantized energy levels, and the spin orientation of such structures is subject to quantum uncertainty ${ }^{14}$. The properties of these magnetic systems can be described by model spin Hamiltonians incorporating Zeeman splitting ${ }^{15}$, magneto crystalline anisotropy $y^{8,9}$ and spinspin interaction ${ }^{6,7}$. As model magnetic structures we investigate individual $\mathrm{Mn}$ atoms and $\mathrm{Mn}$ dimers that are placed on $\mathrm{Cu}$ binding sites of a $\mathrm{Cu}_{2} \mathrm{~N}$ decoupling layer that is grown on a $\mathrm{Cu}(100)$ substrate $^{16}$. This set-up makes the atoms electrically accessible to the probe tip of a scanning tunnelling microscope (STM). The measured current flows between the tip and the $\mathrm{Cu}$ substrate, with the decoupling layer preventing excessive broadening of the $\mathrm{Mn}$ atom's magnetic energy levels. Figure $1 \mathrm{a}, \mathrm{b}$ shows STM images of the structures. A single $\mathrm{Mn}$ atom on $\mathrm{Cu}_{2} \mathrm{~N}$ has a localized spin $S=5 / 2$ and shows only small magnetic anisotropy ${ }^{9}$. In high magnetic fields, this leads to a behaviour similar to the free atomic spin, giving a ladder of quantized spin states (Fig. 1c,d, central column). To determine the effect of spin-polarized tunnel current on the state of the local spin, we developed a technique that allows the measurement of the same structure with spinpolarized and non-spin-polarized electric currents (see the Methods section and Fig. 1).

Figure 1c,d shows differential conductance spectra $\mathrm{d} I / \mathrm{d} V$ recorded with the STM tip positioned over an individual $\mathrm{Mn}$ atom at a magnetic field of $B=7 \mathrm{~T}$ and at $0.5 \mathrm{~K}$. The measurement details are described in the Methods section. The spectrum shows two steps, one at threshold voltage $V_{\mathrm{ex}}=+0.7 \mathrm{mV}$ and one at $-V_{\mathrm{ex}}$. These steps are the result of inelastic electron tunnelling ${ }^{17}$, where tunnelling electrons with sufficient energy promote the Mn from its ground state $(m=+5 / 2, m$ is the magnetic quantum number) to the first excited spin state $(m=+3 / 2)$, which is $0.7 \mathrm{meV}$ higher in energy ${ }^{9}$. A spin-averaging (non-spin-polarized) STM tip gives steps of equal height (Fig. 1c), but a spin-polarized tip gives a larger step for negative $V$ than for positive $V$ (Fig. 1d). This asymmetry is the consequence of a selection rule for spin excitations with tunnelling electrons: total spin angular momentum is conserved during the tunnelling process ${ }^{13,18}$. As this spin excitation changes $m$ for the atom by -1 , it requires down-electron states in the tip when current flows out of the tip $(V>0)$, and up-electron states in the tip when current flows into the tip $(V<0)$ (see Supplementary Fig. S1; refs 18-23). The tip's degree of spin polarization $\eta$ can be determined quantitatively from the ratio of these step heights (see the Methods section). For the tip used in Fig. 1d the polarization is $\eta=0.31 \pm 0.03$ along the direction of the magnetic field.

All inelastic tunnelling events inherently excite the spin system out of the ground state. As a result, these measurements offer access not only to the excitation energies but also to a fundamental timescale: the time required for excited spin states to relax back into the ground state. At low currents, each consecutive tunnelling electron encounters the spin in its ground state because spontaneous relaxation is more frequent than excitation by tunnelling electrons. At sufficiently high currents there is a significant probability for a tunnelling electron to encounter the spin already in an excited state and excitations into even higher states are possible. We make use of the capability of the STM to increase the current independent of the applied voltage by moving the tip closer to the surface. Whereas spectra of a Mn atom probed with a spin-averaging tip do not change when the current is increased (Fig. 1c), we find that the spectrum of the same Mn atom changes markedly as a spin-polarized tip is moved closer to the surface (Fig. 1d). The most prominent change occurs for negative $V$, where the high-current spectrum drops from its peak value near $V=-V_{\text {ex }}$ and declines to a much lower value at large negative $V$. A qualitative explanation for this drop-off is that the time-average occupation of the spin states changes from being mostly in the ground state to being mostly in excited states, and the conductance through the Mn atom changes depending on its spin state. Below, we develop a quantitative model that confirms this explanation.

For a $\mathrm{Mn}$ atom, all spin excitations allowed by the spinconservation rule (including those starting from excited states) have approximately the same energy and therefore all successive

\footnotetext{
IIBM Research Division, Almaden Research Center, San Jose, California 95120, USA, ${ }^{2}$ Institute of Applied Physics, University of Hamburg, 20355 Hamburg, Germany, ${ }^{3}$ Max-Planck-Institut für Festkörperforschung, 70569 Stuttgart, Germany, ${ }^{4}$ Kamerlingh Onnes Laboratorium, Universiteit Leiden, 2300 RA Leiden, The Netherlands. 'Present address: Center for Nanoscale Science and Technology, NIST, Gaithersburg, Maryland 20899, USA. *e-mail: heinrich@almaden.ibm.com.
} 
a
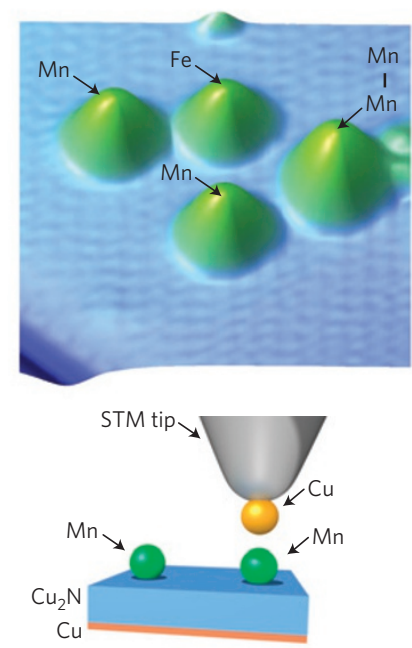

c

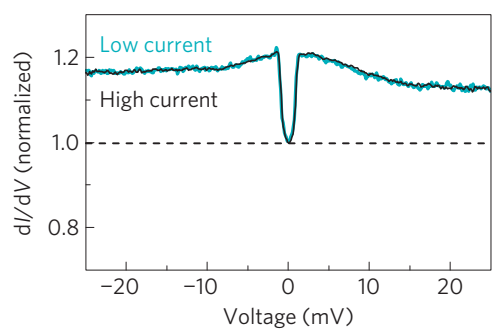

b
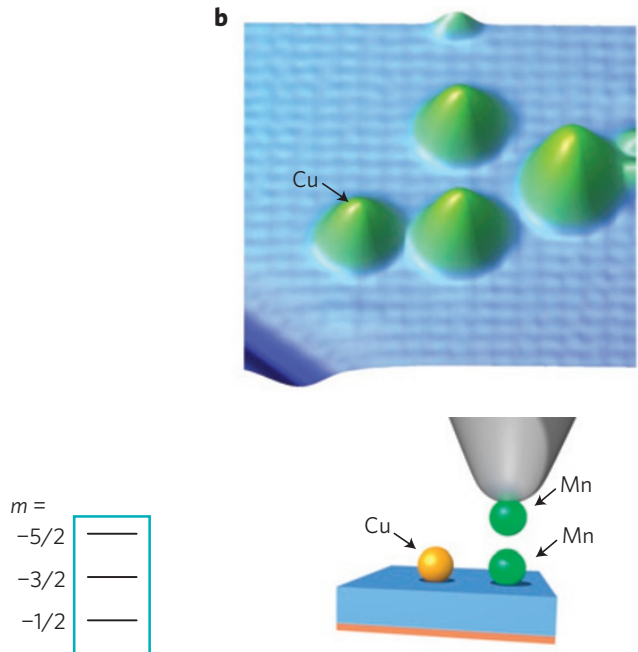

d

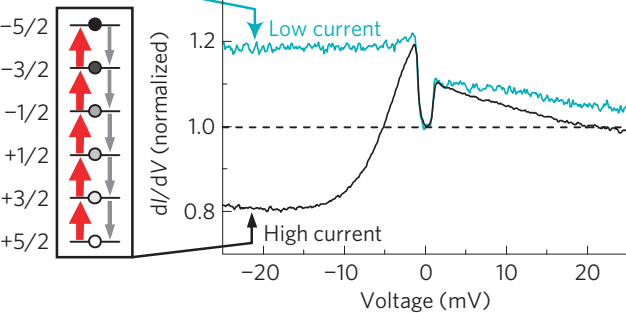

Figure 1 | Spin-averaging and spin-polarized spectra of a $\mathbf{M n}$ atom on a $\mathbf{C u}_{\mathbf{2}} \mathbf{N} / \mathbf{C u}(\mathbf{1 0 0})$ surface. STM images $(6.3 \times 7.5) \mathrm{nm}{ }^{2}, V=-0.5 \mathrm{~V}, I=2 \mathrm{nA}$, colour code: blue (green) is low (high) tip height. Mn atoms appear as $0.28-n m$-tall protrusions and the Mn dimer has an elongated shape ${ }^{6}$. $\mathbf{a}, \mathrm{A}$ Cu-terminated tip serves as a spin-averaging (non-spin-polarized) tip. b. A Mn-terminated tip yields spin-polarization in the tip's electronic density of states (see the Methods section). c, Differential conductance spectra ( $d / / d V$ ) of a Mn atom acquired with the Cu-terminated tip. $\mathbf{d}$, Spectra of the same $\mathrm{Mn}$ atom recorded with the $\mathrm{Mn}$-terminated tip. The spectra in $\mathbf{c}$ and $\mathbf{d}$ are measured at magnetic field $B=7 \mathrm{~T}$ applied perpendicular to the surface. The steps at $V_{\text {ex }}= \pm 0.7 \mathrm{mV}$ indicate the onset of inelastic electron tunnelling that excites the $\mathrm{Mn}$ into higher spin states. Spectra are normalized to unity at $V=0$. The low-current spectra (blue) are recorded at a large tip-sample distance (characterized by the conductance at $V=0: G=0.02 \mu S$ ) and the high current spectrum at a reduced tip-sample distance $(G=0.75 \mu \mathrm{S})$. Spin-state diagrams (central column) schematically show transitions occurring for negative $V$ at low and high spin-polarized currents, respectively.

excitations happen at the same threshold voltage $V_{\text {ex }}$. To experimentally distinguish between spin excitations starting from ground and excited states, we briefly turn to a Mn dimer, which has strong antiferromagnetic coupling between the two atoms so its ground state has zero $\operatorname{spin}^{6}$. In this dimer, successive excitations into higher states require progressively higher energies (Fig. 2a). Figure 2 shows the effect of such spin pumping on the Mn dimer using a spin-averaging tip. At low current the presence of steps in the spectrum at $\pm 7.4 \mathrm{mV}$ indicates the onset of transitions that begin in the dimer's singlet ground state $S=0$ and go to the triplet states $S=1$ (Fig. 2b). These steps occur at a voltage corresponding to the energy $J$ of the exchange coupling between the dimer's Mn atoms ${ }^{6}$. We find that more steps emerge in the spectrum at energies $2 \mathrm{~J}$ and $3 J$ when the tunnel current is increased (Fig. 2c,d), corresponding to excitations to the next higher levels. As no transition with energy $2 J$ exists for excitations directly out of the ground state, the presence of the observed steps unambiguously demonstrates that successive excitations from excited states into even higher states occur with substantial probability in such quantum magnetic structures.

To explain the mechanism of spin pumping for the single $\mathrm{Mn}$ atom we developed a quantitative model that describes the behaviour of the atom's spin under the influence of the tunnel current. We model the probability to find the local spin in any given energy eigenstate, and the rates at which transitions between these states occur; these rates are calculated using a quantum mechanical transition intensity operator that is applied to the combined spin state of the atom and the tunnelling electron (details are given in the Methods section). Fitting this model to the measured spectra gives quantitative agreement (Fig. 3 and Supplementary Fig. S2). We find that the drop-off in the spectrum observed for $V<-V_{\text {ex }}$ with the spin-polarized tip occurs in a voltage range that scales with the rate of tunnelling events: when the tunnel current is doubled by moving the tip closer to the surface, the drop to the limiting level occurs in half the voltage range. The model shows that this drop in the spectrum is due to a change in the spin-state distribution to greater occupation of excited states, which have lower differential conductance. For sufficiently large $V$, the system is asymptotically driven to a limiting spin-state distribution, which does not change with further increases of $V$. Consequently, the differential conductance levels off as well. This limiting distribution is set by the probe tip's degree of spin polarization. Figure $3 \mathrm{c}$ shows that for a spin-polarized tip the $m=-5 / 2$ state becomes most occupied at negative $V$, even though it is the highest in energy. When $V$ is positive, in contrast, the atom's spin is driven towards the ground state $(m=+5 / 2)$. In comparison, large non-spin-polarized currents spread out the state occupation equally for both voltage polarities.

This spin-pumping behaviour shows intriguing similarity to conventional spin-momentum transfer. As in magnetic multilayer devices $^{24}$, the orientation of the local spin can be flipped by applying high spin-polarized current and the direction of current 
a

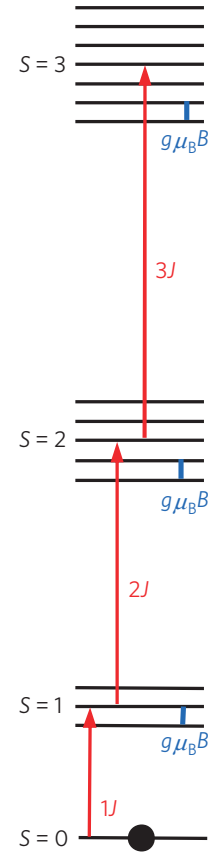

b
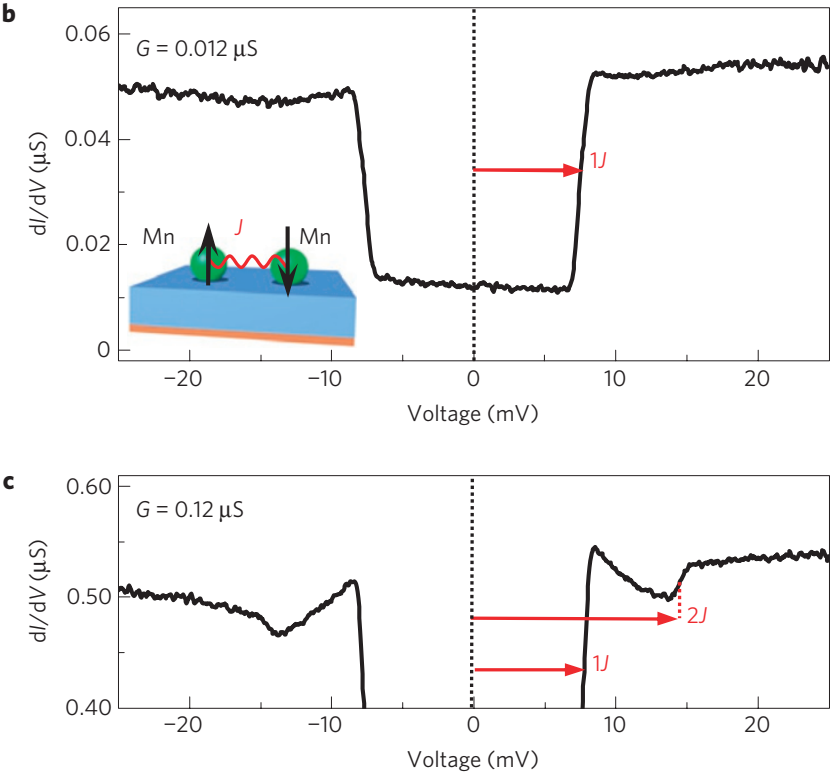

d

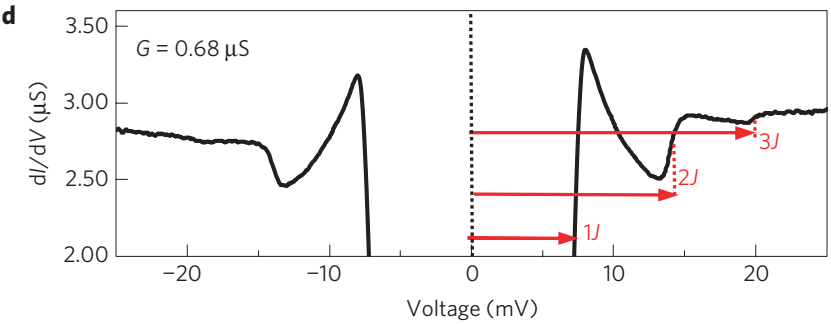

Figure 2 | Spectra of a Mn-Mn dimer. a, The spin-state diagram schematically shows the Mn dimer's spin-state energies (Zeeman splitting not to scale). b, A low-current spectrum $(G=0.012 \mu \mathrm{S}$ ) showing a prominent step at $\pm 7.4 \mathrm{mV}$ corresponding to the transition from the $S=0$ singlet state to the $S=1$ triplet states at exchange coupling energy J. c. When the tunnel current is increased $(G=0.12 \mu \mathrm{S})$, more excitation steps emerge at $\pm 14.6 \mathrm{mV}$ because of the onset of excitations out of $S=1$ into $S=2$ states. $\mathbf{d}$, At further increased tunnel current $(G=0.68 \mu S)$ steps at $\pm 20.1 \mathrm{mV}$ appear. All steps in $\mathbf{b}$-d appear broadened because each multiplet is split by the Zeeman energy $g \mu_{\mathrm{B}} B$, where $B=3 \mathrm{~T}$ applied in-plane and $\mu_{\mathrm{B}}$ is the Bohr magneton. All spectra were obtained with a spin-averaging tip.

flow determines the spatial orientation of the spin (Fig. 3c). Unlike the quantized spin-momentum transfer described here, the magnetization direction is rotated by the transverse component of the spin-polarized current in conventional spin-momentum transfer ${ }^{25}$. The classical magnetization evolves along a definite trajectory from initial to final direction. The quantum spin on the other hand can fully delocalize in the plane perpendicular to the quantization direction, and a longitudinal spin-polarized current can reverse it efficiently. The quantized spin-momentum transfer requires inelastic electron tunnelling in this process, so it has a distinct onset threshold given by the transition energy of the first spin excitation.

The spin-polarized current that causes spin pumping also allows detection of the resulting spin state. There is strong spin-sensitivity in the elastic tunnelling through the atom. This spin-sensitive detection is qualitatively similar to tunnel magnetoresistance devices $^{26}$ and conventional spin-polarized STM where a spindependent density of states in both electrodes describes the conductance ${ }^{13}$. Here, however, the spin-sensitive elastic tunnelling is described by spin filtering of electrons that interact with the Mn spin but do not change their spin state. This elastic spin filtering is well described by the transition intensity function (see the Methods section), and no spin dependence in the density of states in the $\mathrm{Cu}$ substrate is needed. For positive $V$, where the local spin stays aligned with the majority tip states near the Fermi energy (up states), a high elastic conductance through the atom is observed. In contrast, when the Mn spin is pumped into anti-alignment with the tip's spin polarization by applying negative $V$, the up electrons have a low elastic tunnelling probability. This results in a remarkably strong drop-off in the elastic conductance that more than offsets the increase in conductance resulting from the inelastic tunnelling (see Supplementary Fig. S3). Consequently, the measured spectrum develops the distinct drop-off for negative voltages. With a spinaveraging tip, the change in elastic conductance compensates the change in inelastic conductance so the spectrum remains nearly unchanged even at high currents. The model fit to the measured spectra shows that the tunnelling of up electrons is suppressed by a factor of 215 compared with down electrons, when the $\mathrm{Mn}$ spin is in its highest excited state $(m=-5 / 2)$. This suppression corresponds to a spin polarization of 0.99 for elastic tunnelling with the $\mathrm{Mn}$ in this spin state.

To determine the mechanism by which spin relaxation occurs, we analysed the dependence of the spin-pumping spectra on the magnetic field. The spectra at $3 \mathrm{~T}$ show stronger pumping behaviour (faster drop at negative voltage) than the $7 \mathrm{~T}$ spectra (Fig. 4a). We find that this behaviour is well described by modelling the spontaneous relaxation (Fig. $4 \mathrm{~b}$ ) as a spin-scattering process: electrons in the substrate interact with the $\mathrm{Mn}$ by exchanging energy and spin angular momentum, just as the tunnelling electrons do. As these electrons begin and end in the substrate, they do not register as measurable current. This relaxation mechanism is characterized by a single parameter $G_{s}=2.7 \pm 0.2 \mu \mathrm{S}$, which gives the effective interaction of the substrate conduction electrons with the Mn spin. $G_{\mathrm{s}}$ applies independent of magnetic field and Mn spin 
a
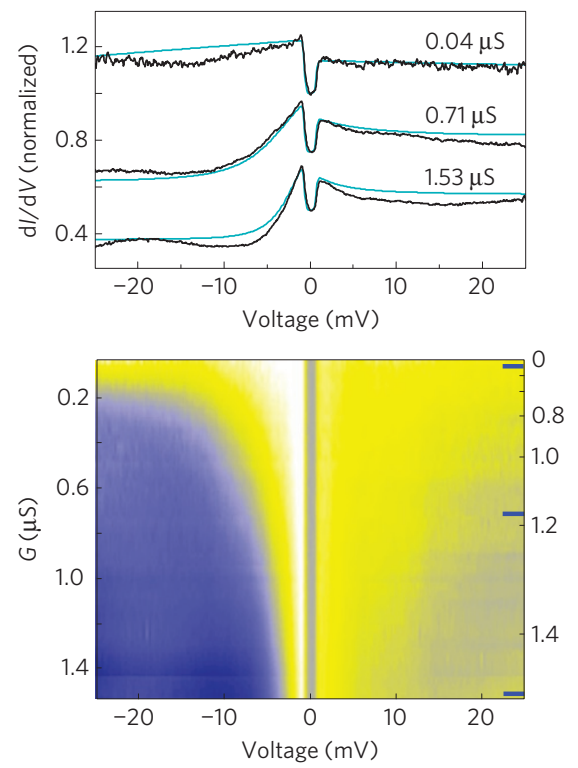

b
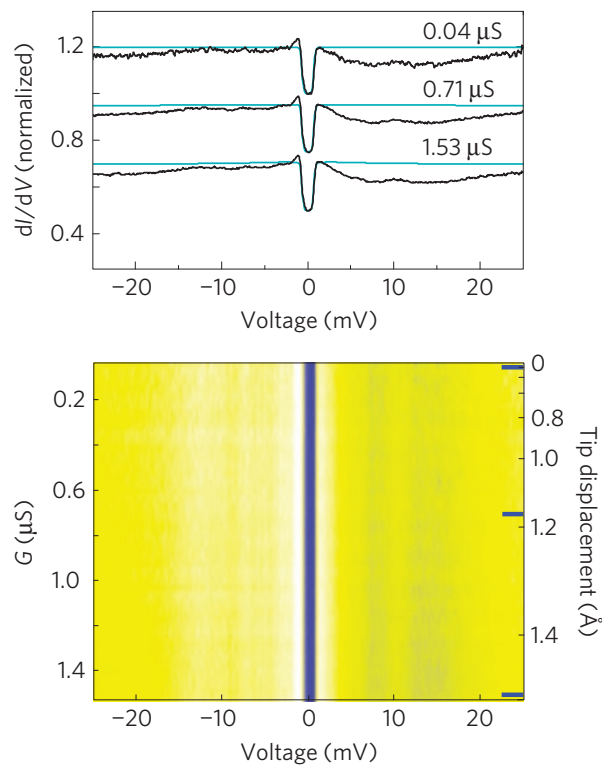

c

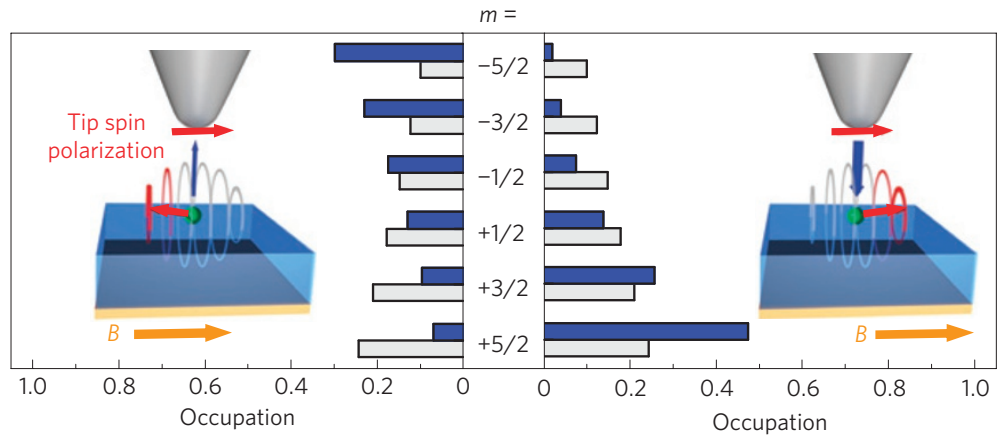

Figure 3 | Mn atom spectra and spin-state occupations during spin pumping. a, Spectra measured on a $\mathrm{Mn}$ atom at $B=7 \mathrm{~T}$ (in-plane direction) with a spin-polarized tip. $\mathbf{b}$, Spectra of the same $\mathrm{Mn}$ atom probed with a spin-averaging tip. The top panels in $\mathbf{a}, \mathbf{b}$ show individual spectra (black), labelled by $G$ (conductance at $V=0$ ) and normalized to unity at $V=0$. Spectra with different $G$ are shifted vertically by -0.3 for clarity. Calculated spectra (blue) result from the spin pumping model. Best fit for parameters: $G_{s}=2.7 \pm 0.2 \mu S, u=1.09 \pm 0.09, \eta=0.24 \pm 0.04$ for $\mathbf{a}$ and $\eta=0.00$ for $\mathbf{b}$. The bottom panels in $\mathbf{a}, \mathbf{b}$ show a full set of spectra in a conductance map, colour code showing low (high) $\mathrm{d} l / \mathrm{d} V$ in blue (yellow). c, Time-average occupation probabilities of the six $\mathrm{Mn}$ spin states for high tunnel current $(G=1.53 \mu \mathrm{S})$ from the spin-polarized tip (blue) and the spin-averaging tip (grey). The left graph is at $V=-25 \mathrm{mV}$; the right graph is at $+25 \mathrm{mV}$. Insets: Schematics of dominant spin alignments of tip spin polarization and $\mathrm{Mn}$ atom spin at $\pm 25 \mathrm{mV}$.
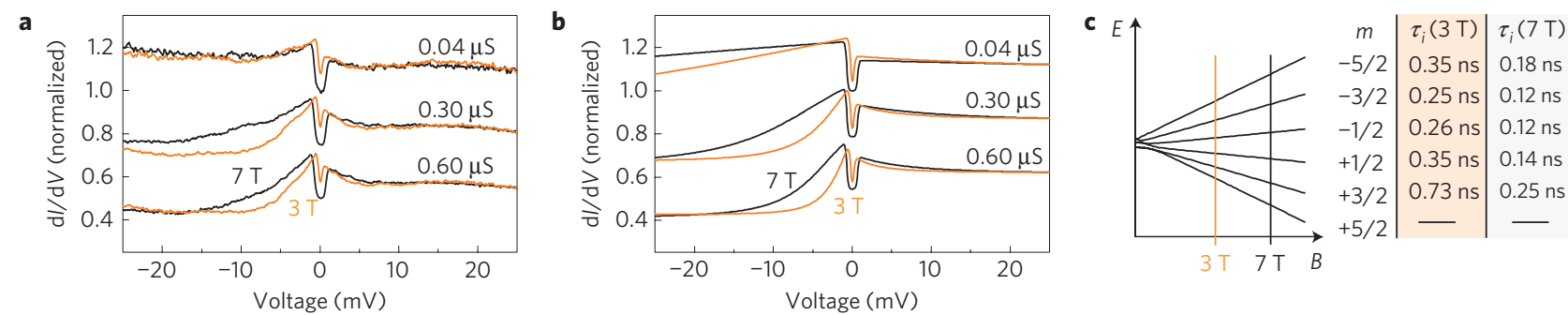

Figure 4 | Spin lifetimes and magnetic-field dependence of Mn atom spectra. a, Comparison of normalized spectra of the same Mn atom measured at in-plane magnetic fields of $3 \mathrm{~T}$ (orange) and $7 \mathrm{~T}$ (black) using the same spin-polarized tip. b. Model results for the spectra in a using identical model parameters as in Fig. 3a except the magnetic field. c, Energy splitting of the six Mn spin states as a magnetic field is applied in the plane of the surface. The table lists the spin lifetimes $\tau_{i}$ at 3 and $7 \mathrm{~T}$ as derived from the model fit to the spectra (see Supplementary Methods).

state, and is kept constant throughout this work, which suggests that it is an intrinsic property of $\mathrm{Mn}$ on this surface. All of the spectra are well described using this relaxation mechanism alone, so other spin-relaxation mechanisms found in quantum magnets, such as spin-phonon coupling ${ }^{27}$ seem to not have a significant role here. In this relaxation mechanism, the number of substrate electrons capable of inducing relaxation out of an excited state scales with the state's excitation energy, so the relaxation rate is proportional to $G_{\mathrm{s}} \cdot V_{\mathrm{ex}} / e$. As the transition energy $V_{\mathrm{ex}}$ for the $\mathrm{Mn}$ atom is mostly given by the Zeeman energy ${ }^{9}$, the spin lifetime should be reciprocal to the magnetic field. Indeed, the spin lifetimes yielded by the model follow this trend: the longest lifetime at $7 \mathrm{~T}$ is $0.25 \pm 0.04 \mathrm{~ns}$ for the first excited spin state, which increases to $0.73 \pm 0.10 \mathrm{~ns}$ at $3 \mathrm{~T}$ (Fig. $4 \mathrm{c}$ ). 
Spin-momentum transfer is a demonstrated method for switching conventional bi-stable magnetic bits ${ }^{1,24}$. Our results show that a similar effect is active in quantum spin systems when the transport electrons have sufficient energy to induce quantum transitions in the localized spin. Spin-momentum transfer could be used for electrical switching of bi-stable quantum spin systems, such as single-molecule magnets ${ }^{2}$. Although the spin lifetimes studied here are too short for practical applications, atomic-scale spin systems are capable of far longer lifetimes ${ }^{12}$. On the basis of the dominant relaxation mechanism found here, more effective decoupling layers might bring the lifetimes of atomic-scale structures into a range suitable for use in quantum information devices, while retaining the advantages of electrical control and sensing.

Note added in proof: In the final stages of manuscript preparation, a similar theoretical description of spin pumping ${ }^{28}$ was posted.

\section{Methods}

Tip preparation. The tip's spin polarization is turned on and off by exchanging the last apex atom of the tip between $\mathrm{Cu}$ and $\mathrm{Mn}$. Starting from a sharp Cu-coated tip (Fig. 1a), the last $\mathrm{Cu}$ atom at the tip apex is transferred to the surface ${ }^{6,29}$ and a magnetic atom is transferred to the tip by the reverse process (Fig. 1b). This leads to a paramagnetic tip that is aligned in the magnetic field ${ }^{30}$. These tips show no pronounced features in reference spectra recorded over the bare $\mathrm{Cu}$ surface and are well characterized by an energy-independent spin-polarized density of states near the Fermi energy. The degree of spin polarization $\eta$ is defined as in ref. 13 and experimentally determined by the height ratio of the conductance steps at $\pm V_{\mathrm{ex}}$ (see Supplementary Fig. S1). The Mn-terminated tips used in this work typically yield spin polarization $\eta \approx 0.2-0.3$ (for $B>2 \mathrm{~T}$ ).

Conductance spectra. All spectra are recorded with the STM tip positioned on top of the $\mathrm{Mn}$ atoms and in the middle of the $\mathrm{Mn}$ dimer as given by the maxima in the topography. We measure the differential conductance $\mathrm{d} I / \mathrm{d} V$ as a function of $V$, where $I$ is the tunnel current and $V$ is the sample voltage. Lock-in detection at $768 \mathrm{~Hz}$ with $50 \mu \mathrm{V}$ modulation amplitude is used. The tunnel current is varied independent of the voltage by changing the tip-sample distance. The conductance $G$ at $V=0$ serves as measure for the conductance of the tunnel junction at any given tip-sample distance.

Spin Hamiltonian. All model calculations are based on the full spin Hamiltonian for $\mathrm{Mn}$ on this surface as described in ref. 9 and in the Supplementary Methods, using parameters $g=1.98, D=-41 \mu \mathrm{eV}, E=7 \mu \mathrm{eV}$, easy axis out-of-plane. For simplicity in the text, we characterize the energy eigenstates by using only the $m$ quantum number.

Model. We use a set of rate equations in the form of the Pauli master equation ${ }^{31}$, in which quantum mechanical transition intensities describe the transition rates. Here we take into account excitations and de-excitations by tunnelling electrons, as well as de-excitations through spontaneous relaxation. Solving the rate equations for the steady-state conditions yields the time-average population of the spin states and the time-average tunnel current as a function of the externally controlled parameters: tip-sample distance, voltage, magnetic field, spin polarization of the tip and temperature. See Supplementary Methods for a detailed description.

The transition intensity function we use is $Y \propto|\langle j|\mathbf{S} \cdot \boldsymbol{\sigma}+u| i\rangle|^{2}$, where $|i\rangle$ and $|j\rangle$ are initial and final spin states of the combined system of tunnelling electron and local spin and $\boldsymbol{\sigma}$ and $\mathbf{S}$ are the corresponding spin vector operators. This function is based on exchange interaction between the tunnelling electrons and the local spin in a manner similar to previous works, ${ }^{9,1922,23}$. We allow $|j\rangle$ to equal $|i\rangle$ to model elastic tunnelling. We introduce the scalar parameter $u$ to characterize the dependence of the elastic transitions in $Y$ on the relative alignment of the local spin and the spin of the tunnelling electron ${ }^{21} . Y$ becomes proportional to that in ref. 22 when $u=1 / 2(S+1)$ and to that in refs 9,19 for $u=0$. We find $u=1.09 \pm 0.09$ to give the best agreement for all $\mathrm{Mn}$ atom spectra independent of all externally controlled parameters (Supplementary Fig. S4 shows more fits).

Received 5 October 2009; accepted 3 February 2010; published online 14 March 2010

\section{References}

1. Chappert, C., Fert, A. \& Van Dau, F. N. The emergence of spin electronics in data storage. Nature Mater. 6, 813-823 (2007).

2. Bogani, L. \& Wernsdorfer, W. Molecular spintronics using single-molecule magnets. Nature Mater. 7, 179-186 (2008).

3. Grose, J. E. et al. Tunnelling spectra of individual magnetic endofullerene molecules. Nature Mater. 7, 884-889 (2008).
4. Park, J. et al. Coulomb blockade and the Kondo effect in single-atom transistors. Nature 417, 722-725 (2002).

5. Liang, W., Shores, M. P., Bockrath, M., Long, J. R. \& Park, H. Kondo resonance in a single-molecule transistor. Nature 417, 725-729 (2002).

6. Hirjibehedin, C. F., Lutz, C. P. \& Heinrich, A. J. Spin coupling in engineered atomic structures. Science 312, 1021-1024 (2006).

7. Chen, X. et al. Probing superexchange interaction in molecular magnets by spin-flip spectroscopy and microscopy. Phys. Rev. Lett. 101, 197208 (2008).

8. Tsukahara, N. et al. Adsorption-induced switching of magnetic anisotropy in a single iron(II) phthalocyanine molecule on an oxidized $\mathrm{Cu}(110)$ surface. Phys. Rev. Lett. 102, 167203 (2009).

9. Hirjibehedin, C. F. et al. Large magnetic anisotropy of a single atomic spin embedded in a surface molecular network. Science 317, 1199-1203 (2007).

10. Thomas, L. et al. Macroscopic quantum tunnelling of magnetization in a single crystal of nanomagnets. Nature 383, 145-147 (1996).

11. Leuenberger, M. N. \& Loss, D. Quantum computing in molecular magnets. Nature 410, 789-793 (2001).

12. Ardavan, A. et al. Will spin-relaxation times in molecular magnets permit quantum information processing? Phys. Rev. Lett. 98, 057201 (2007).

13. Bode, M. Spin-polarized scanning tunnelling microscopy. Rep. Prog. Phys. 66, 523-582 (2003)

14. Gatteschi, D., Sessoli, R. \& Villain, J. Molecular Nanomagnets (Oxford Univ. Press, 2006).

15. Heinrich, A. J., Gupta, J. A., Lutz, C. P. \& Eigler, D. M. Single-atom spin-flip spectroscopy. Science 306, 466-469 (2004).

16. Choi, T., Ruggiero, C. D. \& Gupta, J. A. Incommensurability and atomic structure of $c(2 \times 2) \mathrm{N} / \mathrm{Cu}(100)$ : A scanning tunnelling microscopy study. Phys. Rev. B 78, 035430 (2008).

17. Stipe, B. C., Rezaei, M. A. \& Ho, W. Single-molecule vibrational spectroscopy and microscopy. Science 280, 1732-1735 (1998).

18. Balashov, T. et al. Inelastic electron-magnon interaction and spin transfer torque. Phys. Rev. B 78, 174404 (2008).

19. Fernandez-Rossier, J. Theory of single-spin inelastic tunneling spectroscopy. Phys. Rev. Lett. 102, 256802 (2009).

20. Fransson, J. Spin inelastic electron tunneling spectroscopy on local spin adsorbed on surface. Nano Lett. 9, 2414-2417 (2009).

21. Persson, M. Theory of inelastic electron tunneling from a localized spin in the impulsive approximation. Phys. Rev. Lett. 103, 050801 (2009).

22. Lorente, N. \& Gauyacq, J. P. Efficient spin transitions in inelastic electron tunneling spectroscopy. Phys. Rev. Lett. 103, 176601 (2009).

23. Kirschner, J. Direct and exchange contributions in inelastic scattering of spin-polarized electrons from iron. Phys. Rev. Lett. 55, 973-976 (1985).

24. Myers, E. B., Ralph, D. C., Katine, J. A., Louie, R. N. \& Buhrman, R. A. Current-induced switching of domains in magnetic multilayer devices. Science 285, 867-870 (1999).

25. Slonczewski, J. C. Current-driven excitation of magnetic multilayers. J. Magn Magn. Mater. 159, L1-L7 (1996).

26. Moodera, J. S., Kinder, L. R., Wong, T. M. \& Meservey, R. Large magnetoresistance at room temperature in ferromagnetic thin film tunnel junctions. Phys. Rev. Lett. 74, 3273-3276 (1995).

27. Leuenberger, M. N. \& Loss, D. Spin relaxation in $\mathrm{Mn}_{12}$-acetate. Europhys. Lett. 46, 692-698 (1999).

28. Delgado, F., Palacios, J. J. \& Fernandez-Rossier, J. Spin-transfer torque on a single magnetic adatom. Phys. Rev. Lett. 104, 026601 (2010).

29. Bartels, L., Meyer, G. \& Rieder, K.-H. Controlled vertical manipulation of single Co molecules with the scanning tunneling microscope: A route to chemical contrast. Appl. Phys. Lett. 71, 213-215 (1997).

30. Meier, F., Zhou, L., Wiebe, J. \& Wiesendanger, R. Revealing magnetic interactions from single-atom magnetization curves. Science 320, 82-86 (2008).

31. Kreuzer, H. J. Nonequilibrium Thermodynamics and its Statistical Foundations (Clarendon, 1981).

\section{Acknowledgements}

We thank D. Eigler for mentoring and great discussions, C. Hirjibehedin for suggesting the importance of spin-dependent elastic tunnelling and B. Melior for expert technical contributions. S.L. acknowledges support from the Alexander von Humboldt Foundation; K.v.B. from the German Research Foundation (DFG) (Forschungsstipendium); A.F.O. from the Leiden University Fund; and S.L., M.T., C.P.L. and A.J.H. from the Office of Naval Research.

\section{Author contributions}

All authors conducted the experiments and contributed to the preparation of the paper, S.L., C.P.L. and A.J.H. developed the rate equation model, S.L. and K.v.B. analysed the data.

\section{Additional information}

The authors declare no competing financial interests. Supplementary information accompanies this paper on www.nature.com/naturephysics. Reprints and permissions information is available online at http://npg.nature.com/reprintsandpermissions. Correspondence and requests for materials should be addressed to A.J.H. 